\title{
Analytic analysis of a grid-reinforced asphalt concrete overlay
}

\author{
J. Nielsen \& K. Olsen \\ S\&P Reinforcement Nordic ApS, Odder, Denmark
}

\author{
A. Skar \& E. Levenberg \\ Department of Civil Engineering, Technical University of Denmark, Kgs. Lyngby, Denmark
}

\begin{abstract}
This paper presented an analytic investigation of pavement systems subjected to mill-and-overlay treatments - including grid reinforcement in-between the new asphalt concrete $(\mathrm{AC})$ overlay and the underlying (existing) cracked and aged AC. The investigation was based on an updated version of the classic layered elastic theory capable of handling a fragmented layer. Such a layer mechanically replicates a multi-cracked AC offering considerable vertical stiffness alongside low bending rigidity. A thin high-modulus layer represented the reinforcing grid, fully bonded to the abutting AC layers. Three mill-and-overlay cases and an additional reference case were investigated for a pavement system under the loading of a dual-tire configuration. The cases differed by the milling depth (thin, medium, and thick), and by the inclusion or exclusion of a reinforcing grid. Key responses in the structure and subgrade, commonly associated with different pavement distress, were calculated and compared across the different cases. The analysis suggests that a reinforcing grid can potentially reduce bottom-up cracking and permanent deformation within the AC overlay for the medium and thick mill-and-overlay cases. For the thin mill-and-overlay case, the analysis suggests that topdown cracking is the expected distress mechanism. In this context, the inclusion of a reinforcing grid seemed to be ineffective. Finally, it is found that adding reinforcement to any of the mill-and-overlay cases produces only a marginal effect on key responses linked to the development of permanent deformation deeper in the structure and subgrade.
\end{abstract}

Keywords: Pavement analysis, mill-and-overlay, reinforcement, layered elastic theory, fragmented layer formulation

\section{INTRODUCTION}

Pavement systems serve as the economic and social foundation of every country, with asphalt pavements being the most common type (EAPA and NAPA 2011). These systems continually deteriorate under traffic loadings in combination with weather effects and therefore require regular maintenance. With the increase in traffic loadings (e.g., heavier trucks, platooning), and with climate change effects (i.e., more extreme weather situations, sea rise, prolonged rain periods, higher moisture contents in the unbound layers), maintenance activities often prioritise the improvement of load-carrying capacity (i.e., the time until major repair work is needed) - and not just damage repair and return to pristine conditions. One common maintenance treatment in asphalt pavements is mill-andoverlay (Correia and Bueno 2011). This treatment applies to the full-width of the pavement or the full-width of a lane, and consists of partial-depth milling of the existing - 
aged and damaged - asphalt concrete (AC), and then repaving with new AC. When the paved thickness has (nominally) the same thickness as the milled depth, the original load carrying capacity may be approached; when the paved thickness is larger, the overall structural thickness is increased, and the pavement's load-carrying capacity may be improved. Mill-and-overlay is also often employed to address functional distress, such as poor skid resistance and excessive unevenness.

After a mill-and-overlay, pavement systems consist of new AC that is supported by an aged and damaged AC layer. From a mechanistic standpoint, an aged and damaged AC layer especially if severely cracked - exhibits little bending rigidity and therefore sub-optimal support to the new overlay. A possible approach to tackle this situation is to introduce grid reinforcement at the interface between the existing (aged) $\mathrm{AC}$ and the new overlay. Asphalt grid reinforcements are commonly made of carbon-, glass-, or polymer-fibres which are geometrically arranged in a thin bituminous-coated mesh, and thus offer high in-plane stiffness and tensile strength (Zofka et al. 2017).

The engineering analysis of asphalt pavement systems is currently based on layered elastic theory (LET), wherein each layer is considered a continuum. When it comes to including the effects of densely cracked AC layers in LET, it is a common approach to assign them reduced elastic properties (Mamlouk et al. 1990; Baltzer et al. 2017), even though AC stiffens with age (Bell et al. 1994; Harvey and Tsai 1997; NASEM 2007; Baek et al. 2012). Such approach originates from continuum damage mechanics, where properties are degraded isotropically to represent randomly oriented cracks (Lemaitre and Desmorat 2005). Due to the nature of traffic loadings, in combination with environmental effects, cracking in AC has a preferred vertical orientation - with layers gradually transforming under service into a fragmented state, e.g., alligator cracking and block cracking. Thus, from a mechanistic standpoint, the isotropic modulus reduction approach results in under-estimation of the vertical rigidity and overestimation of the bending rigidity.

To address this shortcoming, Levenberg and Skar (2020) recently proposed an analytic formulation (validated against finite element solutions) for a fragmented layer (FL) that can be incorporated into LET. The idea is to model the FL as a Winkler spring-bed with Pasternaktype shear layer(s) for introducing some interaction between the Winkler springs. The springbed stiffness, characterised by $k$, represents the vertical rigidity of the FL; the Pasternak layer(s), characterised by $G$, produce some bending rigidity for the FL. An additional horizontal spring-bed, characterised by $k_{\mathrm{h}}$, is included in the FL model to represent the ability to transfer parallel-oriented (horizontal) shear stresses.

As for analysing the effects of grid-reinforcement, there is currently no accepted engineering approach. Models based on the Finite Element Method (e.g., Taherkhani and Jalali, 2017), are computationally expensive and mandate a large number of input parameters. Such an approach is unlikely to gain acceptance among practitioners. Nielsen et al. (2020) proposed to treat a reinforcing grid as a thin layer within the LET framework, characterised by: (i) Young's modulus, (ii) Poisson's Ratio, (iii) an effective thickness, and (iv) conditions of bonding with the adjoining AC layers. From this simple consideration, representing a grid reinforcement could be achieved by relatively few input parameters, without adding extra computational demand to the framework.

The objective of the current work is to analyse and generate initial intuition on the expected effects of installing an interlayer reinforcing grid as part of a mill-and-overlay maintenance treatment. This is pursued synthetically (i.e. utilizing a computational model and not measurements from the field), by considering a layered elastic system representing a traditional asphalt pavement consisting of AC layer, unbound granular base layer, and soil subgrade. A FL is employed to describe an existing (damaged and aged) AC after milling, and a thin highmodulus layer is employed to represent the grid-reinforcement. The paper investigates several cases for a dual-tire assembly loading that differ by the milling depth and by the inclusion or exclusion of an interlayer grid reinforcement. In each case, key responses commonly linked to performance in pavement design are calculated and presented in contour plots; response peaks are subsequently identified and compared. A summary of findings and a short discussion are offered at the end. 


\section{CASES FOR ANALYSIS}

Consider a three-layered pavement system with a $150 \mathrm{~mm}$ thick aged and cracked AC layer. This layer rests on an unbound granular base and subbase with a combined thickness of $500 \mathrm{~mm}$. The structure is supported by a subgrade soil extending to a large depth. This pavement is maintained by a mill-and-overlay treatment considering four cases which differ by the milling depth and overlay thickness - see Figure 1. Common to all cases is that the milling depth is equal to the overlay thickness, i.e., returning to the original surface elevation. In Case (a) $30 \mathrm{~mm}$ are milled and repaved, in Case (b) $60 \mathrm{~mm}$ are milled and repaved, in Case (c) $90 \mathrm{~mm}$ are milled and repaved, and in Case (d) the entire aged AC thickness of $150 \mathrm{~mm}$ is milled and repaved.

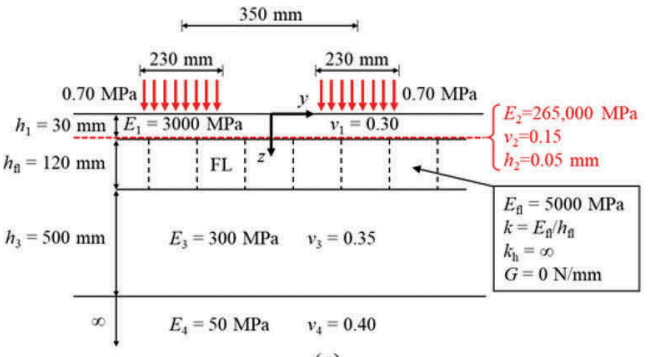

(a)

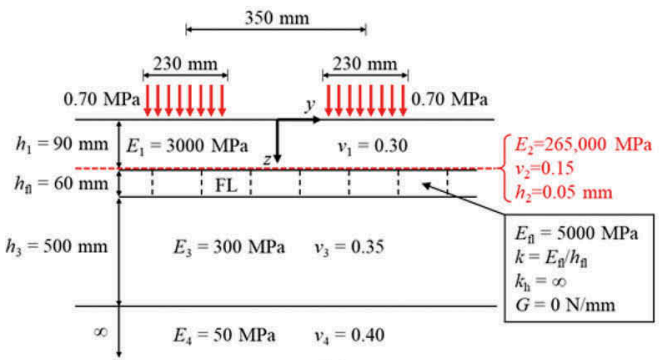

(c)

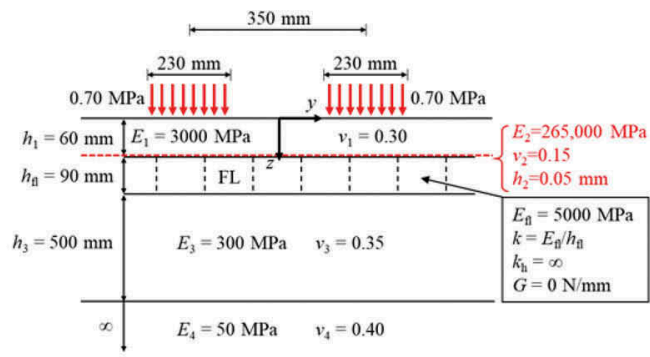

(b)

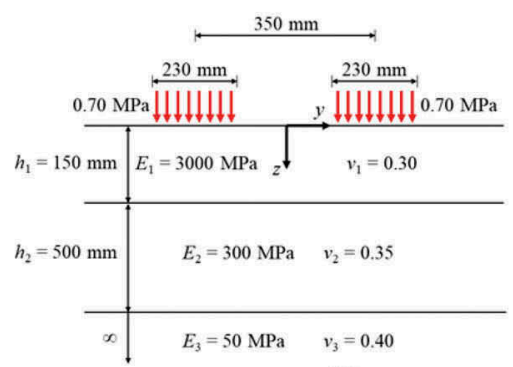

(d)

Figure 1. Cross-sectional view of four pavements for model analysis: (a) $30 \mathrm{~mm}$ mill-and-overlay, (b) $60 \mathrm{~mm}$ mill-and-overlay, (c) $90 \mathrm{~mm}$ mill-and-overlay, and (d) $150 \mathrm{~mm}$ mill-and-overlay.

The pavements in Figure 1 are modelled within the LET framework. A Cartesian coordinate system is considered with its origin located at the surface, and the $z$-axis is oriented towards the subgrade. The model layers are parallel to the $x-y$ plane and extend to infinity. The top layer, representing the AC overlay, has a modulus of $E_{1}=3000 \mathrm{MPa}$ and Poisson's Ratio $v_{1}=0.30$. Any existing (aged) AC is treated as a FL with a Winkler spring-bed stiffness $(k)$ that is equal to a modulus of $E_{\mathrm{fl}}=5000 \mathrm{MPa}$ divided by the FL thickness $h_{\mathrm{fl}}$, a horizontal spring-bed stiffness $k_{\mathrm{h}}$ of infinity, and a Pasternak layer providing zero resistance to shear deformation (i.e., $G=0$ ). The latter assumption corresponds to a densely cracked AC layer with fragments that do not interact. The choice of $5000 \mathrm{MPa}$ to represent an aged AC modulus (and not $3000 \mathrm{MPa}$ as done for the AC overlay) aims to embody age stiffening. The unbound granular materials are represented by a single layer, with a modulus of $E_{3}=300 \mathrm{MPa}$ and a Poisson's Ratio $v_{3}=0.35$. The subgrade soil is treated as a semi-infinite medium with $E_{4}=50 \mathrm{MPa}$ and $v_{4}=0.40$. 
As can be seen in Figure 1, a thin layer representing grid-reinforcement is included for the first three mill-and-overlay cases. This layer resides in-between the top layer and the FL and is assumed to be fully bonded to both. The properties of this layer are directly based on an existing grid product - S\&P Carbophalt ${ }^{\circledR}$ G 200/200 (S\&P 2020). This grid consists of carbon fibres characterised by a modulus of $265 \mathrm{GPa}$ and Poisson's Ratio of 0.15 . The carbon fibres are arranged in square openings, $15 \mathrm{~mm} \times 15 \mathrm{~mm}$ in size, with an average crosssectional area per unit width of the grid of about $50 \mathrm{~mm}^{2} / \mathrm{m}$. Thus, the grid characteristics are smeared based on the volume of fibres per unit area to correspond to an layer thickness of $h_{2}=0.05 \mathrm{~mm}$, modulus of $E_{2}=265,000 \mathrm{MPa}$, and Poisson's Ratio $v_{2}=0.15$. In actuality, the grid is almost $1 \mathrm{~mm}$ thick because of a bituminous material that coats the fibres. This coating contributes to the bonding with the adjacent $\mathrm{AC}$ layers; its contribution to the elastic properties and tensile strength of the grid is considered negligible.

Finally, as shown in Figure 1, all four cases are loaded by two circular areas representing the tire-pavement contact due to a dual-tire assembly. The centres of the circles are located along the $y$-axis, on each side of the coordinate origin with equal offset. The centre-to-centre spacing is $350 \mathrm{~mm}$, and the diameter is $230 \mathrm{~mm}$. Both areas are uniformly loaded by vertical stress with an intensity of $0.7 \mathrm{MPa}$. The above-described characteristics are based on the Danish pavement design guidelines for a standard single-axle load of $120 \mathrm{kN}$ (Baltzer et al. 2017).

\section{ANALYSIS AND RESULTS}

In this section, the first three mill-and-overlay cases in Figures 1a-1c are analysed. The analysis is repeated twice - without and with the inclusion of grid reinforcement. Because Case (d) consists of full removal and repaving of the AC layer, it serves as a reference. Assuming that damage and ageing effects are only confined to the AC layer, Case (d) also represents the pristine mechanical condition of the pavement system. Ultimately, the reinforced cases, unreinforced cases, along with the reference case amount to a total of seven different pavement systems. Calculations were done utilising the LET code ALVA (Skar and Andersen 2020; Skar et al. 2020) that was extended to include the FL formulation from Levenberg and Skar (2020). ALVA is an open-source LET kernel code based on the numerical computing package MATLAB. Soil mechanics sign convention is followed, wherein compressive stresses and strains are positive.

Four key responses are investigated, namely: (i) horizontal strain oriented along the axle direction in the AC overlay $\varepsilon_{y}$; (ii) von Mises stress in the AC overlay $\sigma_{\mathrm{vm}}$; (iii) vertical stress in the aggregate base layer $\sigma_{z}^{\mathrm{b}}$; and (iv) vertical stress in the upper $500 \mathrm{~mm}$ of the subgrade $\sigma_{z}^{\mathrm{sg}}$. The choice to focus on these responses is related to their correlation with cracking and rutting distresses. Specifically, horizontal tensile strains in the AC are closely associated with crack initiation. The von Mises stress in the AC, and the intensity of vertical compressive stresses in the unbound layers and subgrade soil, are all linked to the accumulation of permanent deformation in these materials (Oeser and Möller 2004; Baltzer et al. 2017).

The analysis commenced with a graphical investigation of key response distributions. This was done in the $y-z$ plane for $x=0$ and for $y$ in the range of $\pm 600 \mathrm{~mm}$ ( $z$ is variable). In this context, Figures 2 and 3 present colour-coded contour plots for Case (a) and Case (c) respectively (see Figure 1); these represent a thin overlay situation and a thick overlay situation. Each figure includes four pairs of heat maps, each representing a different key response - one for an unreinforced pavement system (left-hand side) and another for a reinforced pavement system (right-hand side). Peak values of key responses for all four cases are included in Table 1. Note that the colour-coding differs in-between heat map pairs as well as in-between the two figures. To prepare the plots, calculations were performed over a $2 \mathrm{~mm} \times 2 \mathrm{~mm}$ grid in the $y-z$ plane. 

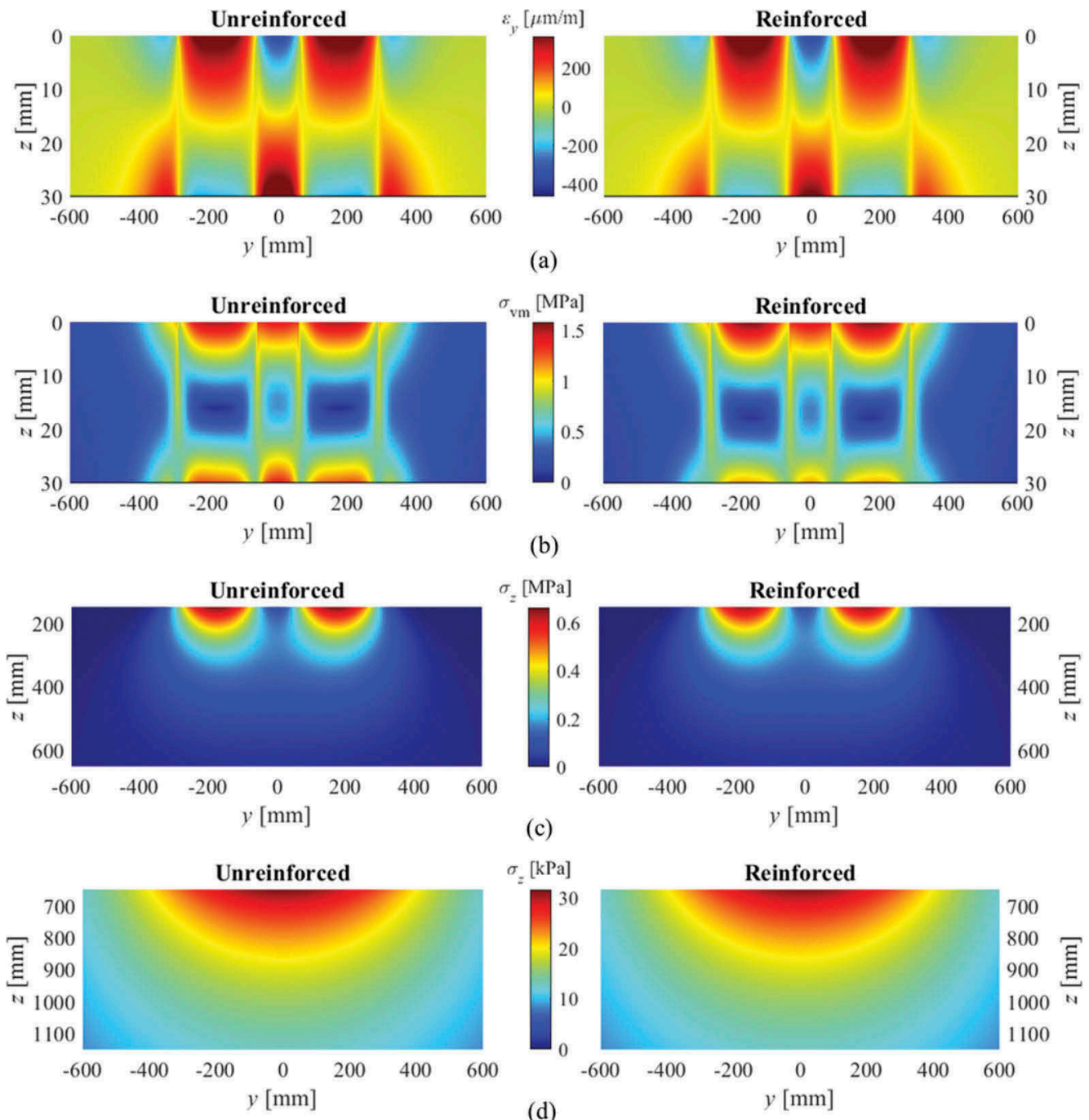

Figure 2. Distributions of the sought key responses in Case (a) of Figure 1: (a) horizontal strain in AC overlay, (b) Von Mises stress in AC overlay, (c) vertical stress in the aggregate base layer, and (d) vertical stress in the subgrade.

Figure 2a presents contour plots of the horizontal strain in $y$-direction within the thin AC overlay, i.e., for the range of $z=0$ to $z=30 \mathrm{~mm}$. In both plots, it can be observed that under the two loading areas, compressive horizontal strains occur at the AC surface while tensile strains occur at the AC bottom. Outside the loading areas, the strain changes sign from compression to tension at the top, and from tension to compression at the bottom. The peak tensile strain is located in-between the two loading areas. While the two contour plots appear visually similar, the introduction of reinforcement causes a slight reduction in the compressive and tensile strain magnitudes at the bottom of the AC overlay, and a slight increase of strains at the AC surface. Figure $2 \mathrm{~b}$ presents contour plots of the von Mises stress within the thin AC overlay. In both plots, it can be observed that two peak stress values occur at the top and bottom - directly under and in-between the two loading areas. Visually, the stress peaks are of slightly larger magnitude at the top. The reinforcement appears to generate a reduction in the von Mises stress at the bottom and a slight increase at the top (compared to the unreinforced situation). Figure $2 \mathrm{c}$ presents contour plots of the vertical stress in the aggregate base layer 
within the range of $z=150 \mathrm{~mm}$ to $z=650 \mathrm{~mm}$. In both plots, it can be seen that stresses are only compressive (positive) with two peaks occurring at the surface - directly under the two loading areas. Visually, the stress distributions appear similar for both the unreinforced and reinforced situations. Lastly, Figure $2 \mathrm{~d}$ presents contour plots of the vertical stress within the subgrade for the depth range $z=650 \mathrm{~mm}$ (i.e., formation level) to $z=1150 \mathrm{~mm}$. In both plots, it can be observed that stresses are only compressive with one peak located at the subgrade surface in-between the two loading areas. Visually, the unreinforced and reinforced situations appear identical.
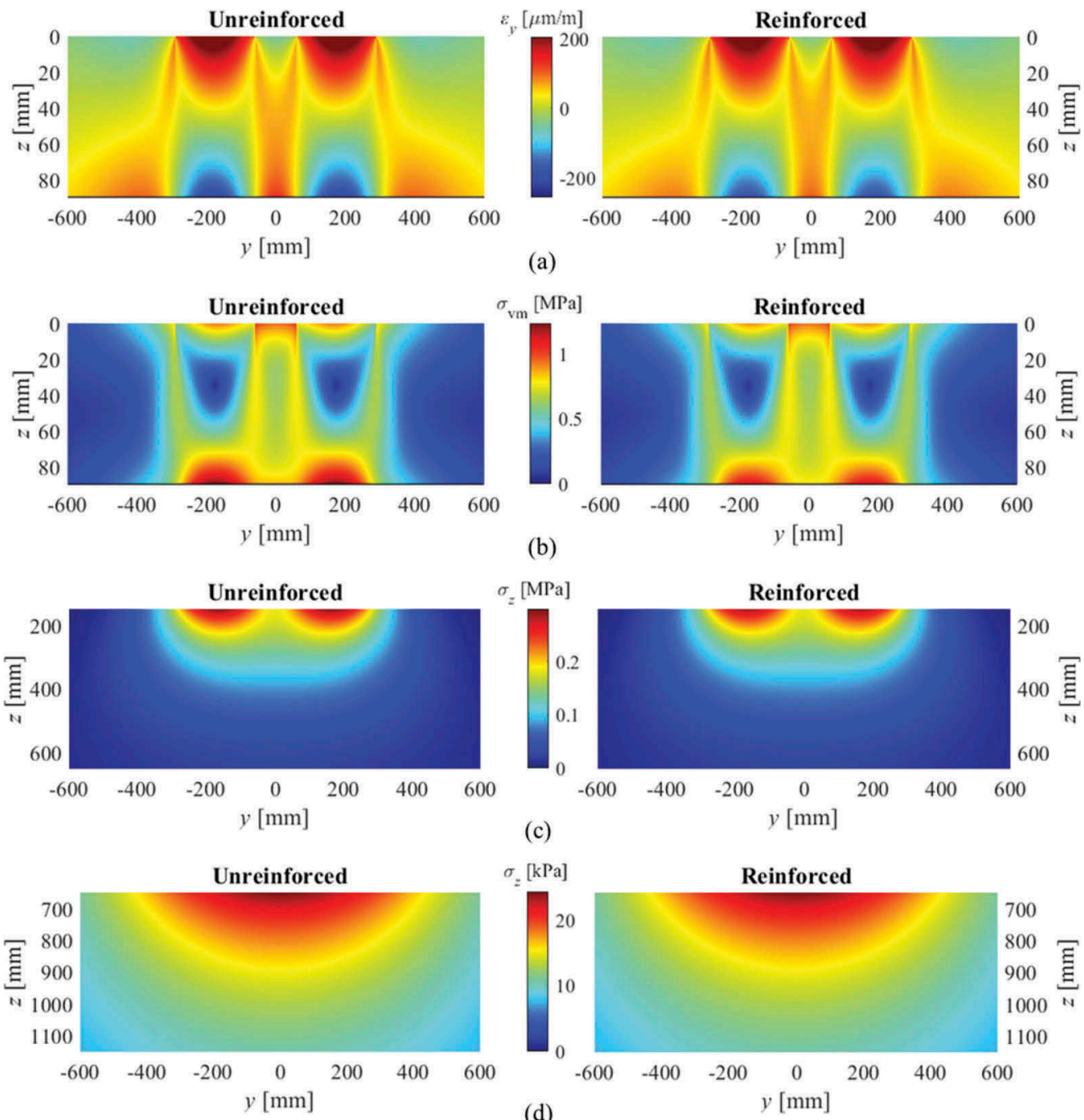

Figure 3. Distributions of the sought key responses in Case (c) of Figure 1: (a) horizontal strain in AC overlay, (b) Von Mises stress in AC overlay, (c) vertical stress in aggregate base layer, and (d) vertical stress in subgrade.

Figure 3a presents contours plots of the horizontal strain in the $y$-direction within the thick AC overlay, i.e., for the range of $z=0$ to $z=90 \mathrm{~mm}$. In both plots, it can be observed that under the two loading areas, compressive horizontal strains occur at the AC surface while tensile strains occur at the AC bottom. Outside the loading areas, the strain changes sign from compression to tension at the top, and from tension to compression at the bottom. Two peak tensile strains are 
identified, each located under one of the two loading areas at the AC bottom. While the two contour plots appear visually similar, the introduction of reinforcement causes a slight reduction in the compressive and tensile strain magnitudes at the bottom of the AC overlay, and a slight increase of strains at the $\mathrm{AC}$ surface. Figure $3 \mathrm{~b}$ presents contour plots of the von Mises stress within the thick AC overlay. In both plots, it can be observed that two peak stress values occur at the bottom - under the two loading areas. The reinforcement appears to generate a reduction in the peak von Mises stress (compared to the unreinforced situation). Figure $3 \mathrm{c}$ presents contour plots of the vertical stress in the aggregate base layer within the range of $z=150 \mathrm{~mm}$ to $z=650 \mathrm{~mm}$. In both plots, it can be seen that stresses are only compressive (positive) with two peaks occurring at the base's surface - under the two loading areas. Visually, the stress distributions appear similar for both the unreinforced and reinforced situations. Lastly, Figure $3 \mathrm{~d}$ presents contour plots of the vertical stress within the subgrade for the depth range $z=650 \mathrm{~mm}$ (i.e., formation level) to $z=1150 \mathrm{~mm}$. In both plots, it can be observed that stresses are only compressive with one peak located at the subgrade surface in-between the two loading areas. Visually, the unreinforced and reinforced situations appear identical.

Contrasting the contour plots of Figure 2 and Figure 3 (i.e., thin overlay vs. thick overlay), several observations can be made. First, concerning the horizontal tensile strain in the AC layer: in the thin overlay case (Figure 2a) the peak value is located at the surface, in-between the two loading areas (for both situations without and with reinforcement); in the thick overlay case (Figure 3a) there are two peaks, both located at the bottom of the AC, each under a loading area (for both situations without and with reinforcement). Second, concerning the von Mises stresses in the AC layer: in the thin overlay case (Figure 2b) there are two peaks, each located at the surface under a loading area (for both situations without and with reinforcement); in the thick overlay case (Figure 3b), there are also two peaks - however, they are both located at the bottom - each under a loading area (for both situations without and with reinforcement). Third, concerning the vertical stresses in the aggregate base layer: in the thin overlay case (Figure 2c) the stress fields at the base surface are highly concentrated under each of the loading areas (for both situations without and with reinforcement); in the thick overlay case (Figure 3c) the stress fields at the base surface are more smeared in-between the loading areas (for both situations without and with reinforcement). Lastly, concerning the vertical stresses at the top of subgrade; stress fields appear similar when contrasting the thin overlay case (Figure 2d) against the thick overlay case (Figure 3d), regardless of the inclusion or exclusion of reinforcement.

The next analysis step utilised the contour plots in Figures 2 and 3, as well as contour plots for Cases (b) and (d) (which are not presented) to identify response peaks across the different mill-and-overlay cases in Figure 1 (with and without reinforcement). The results are summarised in Table 1 for a total of seven situations. The considered peaks are: (i) horizontal tensile (negative) strain in AC overlay $\varepsilon_{y \text {,min }}$, (ii) von Mises stress in $\mathrm{AC}$ overlay $\sigma_{\mathrm{vm} \text {,max }}$, (iii) vertical stress in the aggregate base layer $\sigma_{z, \max }^{\mathrm{b}}$, and (iv) vertical stress in the subgrade $\sigma_{z, \text { max }}^{\text {sg }}$.

Table 1. Peak values of key responses for all four cases in Figure 1 without reinforcement (w/o) and with reinforcement $(\mathrm{w})$.

\begin{tabular}{llllllll}
\hline & \multicolumn{1}{l}{ Case (a) } & & Case (b) & & Case (c) & & Case (d) \\
\cline { 2 - 8 } & w/o & w & w/o & w & w/o & w & w/o \\
\hline$\varepsilon_{\mathrm{y}, \text { min }}[\mu \mathrm{m} / \mathrm{m}$ & -355 & -364 & -260 & -222 & -206 & -181 & -134 \\
$\sigma_{\mathrm{vm}, \max }[\mathrm{MPa}]$ & 1.51 & 1.58 & 1.49 & 1.31 & 1.24 & 1.11 & 0.83 \\
$\sigma_{\mathrm{z}, \max }^{\mathrm{b}}[\mathrm{MPa}]$ & 0.66 & 0.65 & 0.43 & 0.42 & 0.30 & 0.29 & 0.17 \\
$\sigma_{\mathrm{z}, \max }^{\mathrm{sg}}[\mathrm{kPA}]$ & 31.7 & 31.5 & 27.7 & 27.7 & 24.3 & 24.2 & 18.6 \\
\hline
\end{tabular}


First, with respect to the peak tensile strain in the AC overlay: in the thin overlay case, i.e., Case (a), including reinforcement leads to a marginal $2 \%$ increase compared to the unreinforced situation; in the thicker overlay cases, i.e., Case (b) and Case (c), including reinforcement lead to a $15 \%$ and $12 \%$ reduction (respectively) compared to the unreinforced situation. Second, with respect to the peak von Mises stress in the AC overlay: in the thin overlay case, i.e., Case (a), including reinforcement leads to a marginal $4 \%$ increase compared to the unreinforced situation; in the thicker overlay cases, i.e., Case (b) and Case (c), including reinforcement lead to an $11 \%$ and $10 \%$ reduction (respectively) compared to the unreinforced situation. Third, with respect to the peak vertical stress in the aggregate base layer, including reinforcement has a marginal reduction in Case (a), Case (b) and Case (c) compared to the unreinforced situation (respective reduction of $2 \%, 4 \%$, and $3 \%$ ). Fourth, with respect to the peak vertical stress on top of the subgrade, including reinforcement has a negligible effect in Case (a), Case (b) and Case (c) compared to the unreinforced situation. Lastly, the table shows that all four peak responses are lowest for Case (d).

\section{CONCLUSION}

The objective of this work was to provide initial intuition on the effects of introducing interlayer reinforcing grid into a mill-and-overlay maintenance treatment. The analysis was based on a three-layered pavement system subjected to surface loading of a dual-tire assembly, consisting of an AC layer supported by an aggregate base layer resting on a subgrade soil extending to a large depth. The analysis utilised the LET framework to synthetically investigate three mill-and-overlay cases and a reference case. A FL was applied to represent the fully damaged and aged AC layer that was retained in the structure after milling. The reinforcing grid, introduced in-between the FL and the AC overlay, was modelled as a thin high-modulus layer. In each case, horizontal strains and von Mises stresses in the AC overlay, along with vertical stresses in the aggregate base and subgrade were simulated and presented in contour plots for selected cases. Based on these simulations, peaks were identified and contrasted across the different cases.

From this investigation it was found that: (i) for a thick overlay case, the peak tensile strain was located at the bottom - implying bottom-up cracking mode. Adding reinforcement reduced the peak horizontal tensile strain and peak von Mises stress - suggesting potential reduction in cracking and permanent deformation within the overlay; (ii) for a thin overlay case, the peak tensile strain was located at the surface - implying topdown cracking mode. Adding reinforcement marginally increased the peak tensile strain and peak von Mises stress (possibly due to a slight downward shift in the neutral bending axis of the overlay) - suggesting that the reinforcement is ineffective; and (iii) for both thick and thin overlay cases, adding reinforcement has marginal positive effect on the peak vertical stress in the aggregate base layer, and negligible effect on the peak vertical stress in the subgrade.

In studies dealing with grid-reinforced granular base layers, it has been shown that the presence of the grid affects the medium properties in its vicinity - within a so-called transition zone (Luo et al. 2017). This is due to the stress-dependent nonlinear nature of geo-materials, and was not considered in the current analysis. One approach to account for the transition zone within LET framework is to assign an effective (fictitious) modulus to the grid and retain its physical smeared thickness. Such an approach was suggested in the work of Kutay et al. (2020) for grid-reinforced granular base layers, where the modulus within the transition zone was increased. In this context, the grid modulus utilised in this research can be considered as a lower limit value, given that it only represents the actual reinforcement modulus and does not account for the transition zone. In future studies, it is planned to characterise the effective grid modulus based on full-scale experimental investigations. Furthermore, it is anticipated that the effective modulus would appear to be rate- and temperature-sensitive, corresponding to the mechanical nature of AC mixtures. 


\section{REFERENCES}

Baek, C., Underwood, B. S., Kim, Y. R., 2012. Effects of Oxidative Aging on Asphalt Mixture Properties. Transportation Research Record, Vol. 2296(1), pp. 77-85. https://doi.org/10.3141/2296-08

Baltzer, S., Tønnesen, P., Gleerup, S., Holst, M. L., \& Thorup, C, 2017. Design of pavements and reinforcement layers. Vejregler - Danish Road Directorate, Ministry of Transport, Building and Housing.

Bell, C. A., Wieder, A. J., and Fellin., M.J., 1994. Laboratory aging of asphalt-aggregate mixtures: field validation. Washington, DC: Strategic Highway Research Program, National Research Council.

Correia, N. d. S and Bueno, B.d. S., 2011. Effect of bituminous impregnation on nonwoven geotextiles tensile and permeability properties. Geotextiles and Geomembranes, vol. 29, no. 2, pp. 92-101. https://doi. org/10.1016/j.geotexmem.2010.10.004

EAPA and NAPA, 2011. The asphalt paving industry a global perspective. Third Edition

Harvey, J., and B. W. Tsai., 1997. Long-Term Oven Aging Effects on Fatigue and Initial Stiffness of Asphalt Concrete. Transportation Research Record. Vol. 1590(1), pp. 89-98.

Kutay, M. E., Hasnat, M., and Levenberg, E., 2020. Layered Nonlinear Cross Anisotropic Model for Pavements with Geogrids. Submitted for proceedings of Advances in Materials and Pavement Performance Prediction, Aug 3-7.

Lemaitre, J. and Desmorat, R., 2005. Engineering Damage Mechanics. Springer-Verlag Berlin Heidelberg. https://doi.org/10.1007/b13888210

Levenberg, E. and Skar, A., 2020. Analytic pavement modeling with a fragmented layer. International Journal of Pavement Engineering, 1-13. https://doi.org/10.1080/10298436.2020.1790559

Luo, R., Gu, F., Luo, X., Lytton, R. L., Hajj, E. Y., Siddharthan, R. v., Elfass, S., Piratheepan, M., Pournoman, S., 2017. Quantifying the Influence of Geosynthetics on Pavement Performance. Transportation Research Board. https://doi.org/10.17226/24841

Mamlouk, M., Zaniewski, J., Houston, W. and Houston, S., 1990. Overlay design method for flexible pavements in Arizona. Transportation research record, Vol. 1286, pp. 112-122.

National Academies of Sciences, Engineering, and Medicine (NASEM), 2007. Environmental Effects in Pavement Mix and Structural Design Systems. Washington, DC: The National Academies Press. https://doi.org/10.17226/23244

Nielsen, J., Olsen, K., Levenberg, L., and Skar, A., 2020. Fleksible belagninger med asfaltarmering Mekanisk beregningsmodel. Trafik og Veje - April, pp.15-18. (In Danish)

Oeser, M., and Möller, B, 2004. 3D constitutive model for asphalt pavements. International Journal of Pavement Engineering, 5(3), 153-161. https://doi.org/10.1080/10298430412331314281

S\&P (2020), S\&P Carbophalt $®$ G 200/200 Pre-bituminised asphalt reinforcement. https://www.sp-reinforce ment.eu/sites/default/files/field_product_col_doc_file/tds_carbophalt_g_200200_eu_en_0.pdf (date accessed October 7, 2020).

Skar, A. and Andersen, S., 2020. ALVA: An adaptive MATLAB package for layered viscoelastic analysis. Journal of Open Source Software, Vol. 5(52), 2548. https://doi.org/10.21105/joss.02548

Skar, A., Andersen, S., and Nielsen, J., 2020. Adaptive Layered Viscoelastic Analysis (ALVA). Technical University of Denmark. Software. https://doi.org/10.11583/DTU.12387305

Taherkhani, H. and Jalali, M., 2017. Investigating the performance of geosynthetic-reinforced asphaltic pavement under various axle loads using finite-element method. Road Materials and Pavement Design, Vol. 18(S5), pp. 1200-1217. https://doi.org/10.1080/14680629.2016.1201525

Zofka, A., Maliszewski, M., and Maliszewska, D., 2017. Glass and carbon geogrid reinforcement of asphalt mixtures. Road Materials and Pavement Design, Vol. 18(S1), pp. 471-490. https://doi.org/ $10.1080 / 14680629.2016 .1266775$ 